\title{
MILP-Based Dynamic Efficiency Scheduling Model of Battery Energy Storage Systems
}

\author{
Yong-Gi Park*, Chang-Wan Kim** and Jong-Bae Park ${ }^{\dagger}$
}

\begin{abstract}
This paper presents a methodology to determine an optimal operation schedule of a battery energy storage system (BESS) considering dynamic charging/discharging efficiencies considering the output power levels. A novel optimization problem is formulated based on the mixed integer linear programming (MILP) addressing a non-linear charging/discharging efficiency function by a stair-wise linear model. Technical constraints of BESS are also included in the MILP formulation. Test simulation results of BESS operation for the two Korean power systems show the effectiveness of the dynamic efficiency scheduling model compared to the conventional average efficiency ones.
\end{abstract}

Keywords: Battery energy storage system (BESS), Dynamic efficiency scheduling model (DESM), Mixed integer linear programming (MILP), Optimal operation schedule.

\section{Introduction}

Energy storage systems (ESSs) are applicable in various power system fields such as peak shaving, energy arbitrage as a demand response resource, renewable integration, transmission congestion relief, frequency regulation, etc. Recently, in particular, grid-connected battery ESS (BESS) has the opportunity to enter into the practical power systems by showing the tendencies of technological advance and price decrease. Therefore, it can replace the conventional pumped storages which are considered as the costly efficient storages but restricted by the geographical and environmental conditions [1].

ESS can be used by a system operator for peak shaving or load leveling [2,3], and by owner of ESS for the arbitrage trading in response to energy market prices [4]. The applications of energy time shift of BESS with intermittent renewables such as photovoltaics and wind turbines are presented in [5-8]. In particular, [6] and [7] suggested the methodologies to determine the optimal size of ESS considering the scale of the installed renewable capacity. M. S. Kandil et al. simulated optimal power flow containing ESSs in the test grids and analyzed the impact on transmission congestion relief by ESSs [9]. In the customer side, operational strategies of BESS are scheduled to minimize the electric bill in [10] and [11]. Optimal allocation of distributed resources in a microgrid has been resolved in [12] and [13].

Optimization models for determining optimal BESS

$\dagger$ Corresponding Author: Dept. of Electrical Engineering, Konkuk University, South Korea. (jbaepark@konkuk.ac.kr)

* Dept. of Electrical Engineering, Konkuk University, South Korea. (\{draco98,jhroh\}@konkuk.ac.kr)

** Dept. of Mechanical Design and Production Engineering, Konkuk University, South Korea. (draco98@konkuk.ac.kr)

Received: February 1, 2016; Accepted: March 1, 2016 strategies described in previous studies are based on the average constant charging and discharging efficiency values. In practice the efficiency of BESS during charging or discharging is dependent on the internal resistances in the battery cell, and the efficiency of BESS is a function as output power levels under charging or discharging [14]. When a BESS is under charging or discharging at a low C-rate status, it has a high efficiency. The efficiency monotonously decreases when the output power continuously increases. Thus, when the BESS is utilized as an application for peak shaving, its efficiency affects the contribution of the peak load reduction. The efficiency may also influence to the profit when the BESS is used for energy arbitrage application. To reduce the errors between the prior schedules and real operation results of BESS, it is necessary to develop more accurate methodology considering the dynamic BESS efficiency based on the power output levels under charging and discharging.

This paper presents an advanced optimal operation model of BESS considering multi efficiencies under charging and discharging. It is defined as the dynamic efficiency scheduling model (DESM) compared to the conventional average efficiency scheduling model (AESM). Stair-wise functions are introduced in order to approximate nonlinear charging and discharging efficiency curves as the linear models.

This paper is composed as follows: Section 2 describes an efficiency function of BESS of charging and discharging power. Also, we suggest a methodology to address the linearized dynamic efficiency curves. Section 3 introduces the formulation of optimization problems for operating BESS based on the mixed integer linear programming. The results obtained by DESM and AESM are compared in Section 4. Conclusion is given in Section 5. 


\section{Efficiency Curves of BESS}

Fig. 1 shows the simplified diagram of a BESS connected to power system. BESS losses are from power conditioning system (PCS) and battery. PCS loss is associated with the inverter efficiency. The battery loss is varied by the internal resistance which is related with the state of charge, internal and external temperature due to operating duration time [15]. If inverter AC-DC efficiency is fixed and the temperature in battery cell is constantly well managed, voltage and internal resistance of BESS are the functions of the state of charge. In the ideal case, voltage and resistance are not dependent on state of charge. Then charged and discharged energies are described as followings where the leakage resistance is zero [14].

$$
\begin{aligned}
& e_{c}=1.5-0.5 \sqrt{1+p} \\
& e_{d}=0.5+0.5 \sqrt{1-p}
\end{aligned}
$$

Eq. (1) and (2) are dimensionless charged and discharged energy functions of the levelized power respectively, where $e_{c}=E_{C} / Q_{0} V_{0}, e_{d}=E_{d} / Q_{0} V_{0}, p=4 R_{0} P / V_{0}^{2}$, while $Q_{0}$, $V_{0}$ and $R_{0}$ are respectively idealized charge, voltage, and internal resistance of a battery which are independent on the state of charge. $P$ is charging or discharging power of the battery. Assuming the PCS efficiency is a constant,

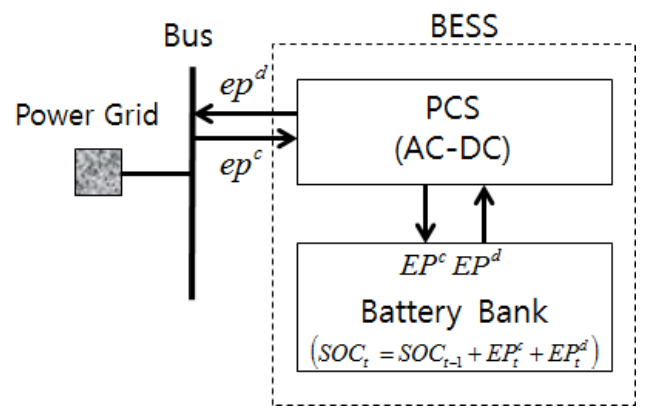

Fig. 1. Simplified diagram of a grid with BESS

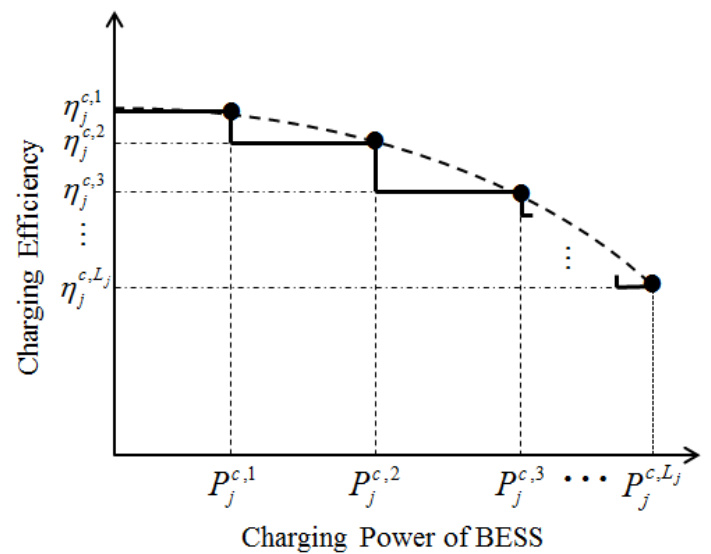

Fig. 2. An approximated stair-wise efficiency function of a BESS. one-way efficiency of BESS under charging or discharging is determined by the efficiency of the battery bank. Additionally, in practice, taking into account other factors that affect the internal resistance of battery, the absolute value of the slope of the efficiency curve become larger, then the efficiency more steeply falls as the power increases.

As described above, the efficiency of BESS is expressed as a non-linear function of charging and discharging power which is dependent on internal resistance of battery. To consider dynamic efficiency model and maintain the linear based optimization formulation, we have introduced a discrete function as shown in Fig. 2. The dashed line is an example of Li-ion battery system charging efficiency function, which is a monotonously decreasing curve corresponding to power levels as Eq. (1). And discharging efficiency curve is similar to charging model as Eq. (2). Each can be approximated by a stair-wise function. It is more accurate curve in close to the origin function if the number of power levels is increased.

\section{Optimal Scheduling Problem of BESS}

\subsection{Objective functions}

BESS can be used for short-term energy time-shift applications by an hour (or 30, 15-minute etc.) basis. The major objectives of optimal BESS schedules will be peak shaving or energy arbitrage. To express based on linear forms, the object functions are formulated as follows:

\subsubsection{Peak shaving}

The aim of peak shaving is to minimize the peak load during the schedule period as (3). Inequality constraint (4) ensures the maximum load among the adjusted loads as Eq. (5) by BESS.

$$
\begin{gathered}
\operatorname{Min} \sum_{t=1}^{T} D^{\max } \\
D^{\max } \geq D_{t}{ }^{\prime}, \quad \forall t \\
D_{t}{ }^{\prime}=D_{t}+\sum_{j=1}^{N}\left[e p_{j, t}^{c}-e p_{j}^{d}\right], \quad \forall t
\end{gathered}
$$

\subsubsection{Energy arbitrage}

The objective of energy arbitrage will be the profit maximization or cost minimization in a perspective of owner or investor of a BESS. In this paper, the objective function is formulated as cost minimization to respond to energy prices as Eq. (6) with the constraint of (5).

$$
\operatorname{Min} \sum_{t=1}^{T} R_{t} \cdot L_{t}{ }^{\prime}
$$




\subsection{Linearization of BESS Constraints}

\subsubsection{External and internal charging/Discharging power}

$$
\begin{gathered}
e p_{j, t}^{c}=\sum_{\ell=1}^{L_{j}}\left(1 / \eta_{j}^{c, \ell}\right) \cdot q_{j, t}^{c, \ell}, \forall t, \forall j \\
e p_{j, t}^{d}=\sum_{m=1}^{M_{j}} \eta_{j}^{d, m} \cdot q_{j, t}^{d, m}, \forall t, \forall j \\
E P_{j, t}^{c}=\sum_{\ell=1}^{L_{j}} q_{j, t}^{c, \ell}, \forall t, \forall j \\
E P_{j, t}^{d}=\sum_{m=1}^{M_{j}} q_{j, t}^{d, m}, \forall t, \forall j
\end{gathered}
$$

Constraints (7) and (8) determine external side charging and discharging power levels of BESS $j$ corresponding to their efficiencies while (9) and (10) are the internal powers under charging and discharging respectively.

\subsubsection{Charging/Discharging phase constraints}

$$
\begin{array}{ll}
v_{j, t}^{c}=\sum_{\ell=1}^{L_{j}} v_{j, t}^{c, \ell}, & \forall t, \forall j \\
v_{j, t}^{d}=\sum_{m=1}^{M_{j}} v_{j, t}^{d, m}, & \forall t, \forall j \\
v_{j, t}^{c}+v_{j, t}^{d} \leq 1, & \forall t, \forall j
\end{array}
$$

Constraints (11) and (12) respectively ensure to select the only one of binary variables of efficiencies corresponding to charging and discharging power level at each time interval. Constraint (13) determines one status of BESS among charging, discharging, and idle at each time interval.

\subsubsection{Charging/Discharging limits}

$$
\begin{gathered}
v_{j, t}^{c, \ell} \cdot \underline{q_{j}^{c, \ell}} \leq q_{j, t}^{c, \ell} \leq v_{j, t}^{c, \ell} \cdot \overline{q_{j}^{c, \ell}}, \quad \forall t, \forall j, \forall m \\
v_{j, t}^{d, m} \cdot \underline{q_{j}^{d, m}} \leq q_{j, t}^{d, m} \leq v_{j, t}^{d, m} \cdot \overline{q_{j}^{d, m}}, \quad \forall t, \forall j, \forall m
\end{gathered}
$$

Constraints (14) and (15) bound charging and discharging range of each power level of BESS- $j$ at time interval- $t$.

\subsubsection{SOC(State of Charge) limits}

$$
\begin{aligned}
S O C_{j}^{\min } & \leq S O C_{j, t} \leq S O C_{j}^{\max }, \forall j, t=1, \ldots, T-1 \\
S O C_{j, T}^{l o w} & \leq S O C_{j, T} \leq S O C_{j, T}^{u p p e r}, \forall j, t=T \\
S O C_{j, t} & =S O C_{j, t-1}+\sum_{\ell=1}^{L_{j}} q_{j, t}^{c, \ell}-\sum_{m=1}^{M_{j}} q_{j, t}^{d, m} \\
& =S O C_{j}^{o}+\sum_{k=1}^{t} \sum_{\ell=1}^{L_{j}} q_{j, k}^{c, \ell}+\sum_{k=1}^{t} \sum_{m=1}^{M_{j}} q_{j, k}^{d, m}, \forall t, \forall j
\end{aligned}
$$

Table 1. Input data for case studies

\begin{tabular}{c|c|c}
\hline \multirow{3}{*}{$\begin{array}{c}\text { System } \\
\text { data }\end{array}$} & Applied Grids & $\begin{array}{c}\text { Main Land and Jeju Island } \\
\text { of South Korea }\end{array}$ \\
\cline { 2 - 3 } & $\begin{array}{c}\text { System Demands and Energy } \\
\text { Prices }\end{array}$ & $\begin{array}{c}\text { Historical MCPs in 2013 } \\
\text { by KPX }\end{array}$ \\
\hline \multirow{4}{*}{$\begin{array}{c}\text { BESS } \\
\text { data }\end{array}$} & Battery Type & Li-ion battery \\
\cline { 2 - 3 } & Rated Capacity & $40 \mathrm{MWh}$ \\
\cline { 2 - 3 } & Rated Power & $20 \mathrm{MW}$ \\
\cline { 2 - 3 } & Depth of Discharge & 0.95 \\
\cline { 2 - 3 } & $\begin{array}{c}\text { One-way Average Efficiency in } \\
\text { Battery Bank }\end{array}$ & 0.970 \\
\cline { 2 - 3 } & $\begin{array}{c}\text { One-way Dynamic Efficiency } \\
\text { Range in Battery Bank }\end{array}$ & 0.952 \\
\cline { 2 - 3 } & Number of Operation Cycle & 0.980 to 0.923 \\
\hline
\end{tabular}

Constraint (16) ensures the minimum and maximum state of charge boundaries of BESS- $j$ at time interval- $t$. It is possible to control the final state of charge range by constraint (17). Constraint (18) determines the state of charge of BESS- $j$ at interval- $t$ using the initial value.

\subsubsection{Total charging/Discharging energy limits}

$$
\begin{aligned}
& \sum_{t=1}^{T} \sum_{\ell=1}^{L_{j}} q_{j, t}^{c, \ell} \leq \text { Cycle }_{j} \cdot\left(S O C_{j}^{\max }-S O C_{j}^{\min }\right), \quad \forall j \\
& \sum_{t=1}^{T} \sum_{m=1}^{M_{j}} q_{j, t}^{d, m} \leq \text { Cycle }_{j} \cdot\left(S O C_{j}^{\max }-S O C_{j}^{\min }\right), \quad \forall j
\end{aligned}
$$

One of the expressions for lifetime of a BESS is cycle life which is the number of cycles for charging and discharging according to depth of discharge [16]. Therefore, we will limit the number of the round-trip operating cycles for a BESS. In this paper, we define one cycle as the one that the cumulated energy of each charge and discharge is equal to the capability of a BESS. Then it can be expressed as the constraints of (19) and (20).

\section{Case Studies}

In this section, comparing with AESM, the proposed DESM is tested in two real-world power systems, Main Land and Jeju Island of South Korea. The two power grids are interconnected via HVDC lines each other and operated by the same independent system operator and market operator, Korea Power Exchange (KPX). The energy spot market clearing prices (MCPs) are determined based on the zonal supply and demand. Considered system demands and MCPs in 2013 are provided by KPX. [17].

The tested Li-ion BESS includes $40 \mathrm{MWh}$ rated capability, 20 MW rated power, and $95 \%$ depth of discharge. Assumptions both charging and discharging efficiencies are considered as the same ones. One-way efficiency of the BESS is divided into 20 pieces from 0.95 to 0.89 


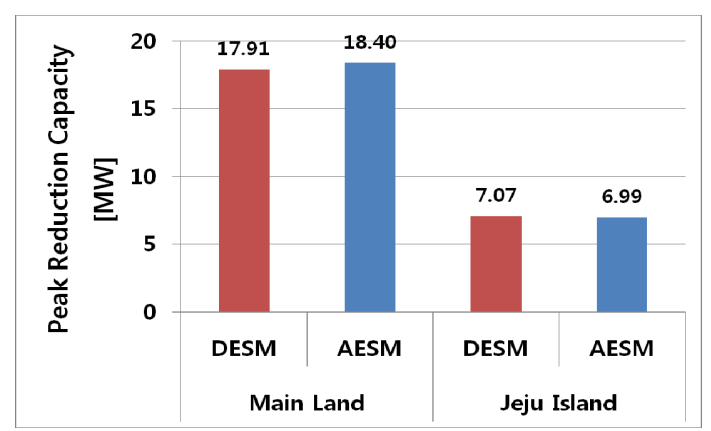

Fig. 3. Contribution of BESS in the peak shaving

corresponding to power levels as the stair-wise function in Fig. 2. The average efficiency of the BESS is assumed as 0.92. Also, it is assumed that the average efficiencies of AC-DC or DC-AC inverter in PCS are to be 0.97 [18].

The algorithms of LP-based AESM and developed MILP-based DESM are implemented in CPLEX 12.6 which is a robust solver for optimization problems.

\subsection{Case-I: Peak shaving application}

In the system operator perspective, the peak shaving application of a BESS can be applied during peak hours of a year. It can be also used to manage peak loads of the electric customers for the purpose of the demand charge savings. In this case, the results of operation schedules of BESS at the grid operator point of view by AESM and DESM models for the peak day in 2013 are compared. They are applied to Main land as well as Jeju Island power systems.

Fig. 3 shows the results of peak shaving by the two scheduling models on the peak days in the two systems. Contribution capacities for peak reduction by DESM and AESM are 17.91 and 18.40 MW in Main Land and 7.07 and 6.99 MW in Jeju Island respectively. In practice, these variations can be understood as the errors of AESM which is a simplified algorithm compared to DESM.

\subsection{Case-II: Energy arbitrage application}

\subsubsection{Scheduled patterns of BESS by DESM and AESM}

Fig. 4 shows the results of BESS operation schedule by DESM and AESM based on daily average MCPs in summer season of 2013. Charging and discharging schedules by DESM are widely distributed on the time horizon to maintain high efficiency with low C-rate while those of AESM are concentrated in two hours. In Jeju Island case, DESM only schedules on the high efficiency sections of BESS, however, AESM is unable to schedule because the difference between maximum and minimum MCPs is small to overcome the loss of BESS with round-trip average efficiency.

Fig. 5 presents the operation results of BESS by DESM
Table 2. Summary of operation results by AESM and DESM of Main Land and Jeju Island

\begin{tabular}{c|c|c|c|c|c}
\hline \multicolumn{2}{c|}{ Power Grid } & \multicolumn{2}{c|}{ Main Land } & \multicolumn{2}{c}{ Jeju Island } \\
\hline \multicolumn{2}{c|}{ Operation Model } & AESM & DESM & AESM & DESM \\
\hline \multicolumn{2}{c|}{ Total Scheduled Days } & 213 & 284 & 109 & 211 \\
\hline \multirow{2}{*}{ Average Power [MW] } & Charge & 14.86 & 5.87 & 13.19 & 3.94 \\
\cline { 2 - 6 } & Discharge & 5.40 & 3.11 & 13.52 & 3.95 \\
\hline $\begin{array}{c}\text { Ave. Frequency } \\
\text { per an operating day }\end{array}$ & Charge & 2.85 & 6.38 & 2.67 & 5.46 \\
\cline { 2 - 6 } & Discharge & 6.54 & 10.58 & 2.17 & 4.73 \\
\hline
\end{tabular}

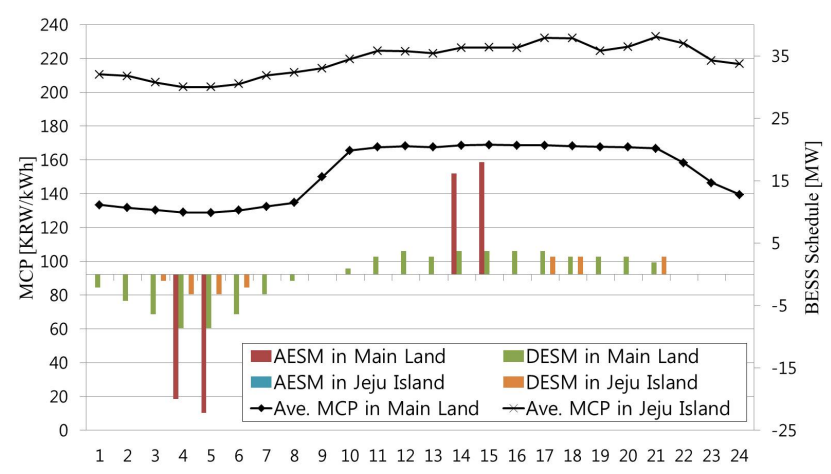

Fig. 4. BESS schedule for the energy arbitrage based on daily average summer MCPs

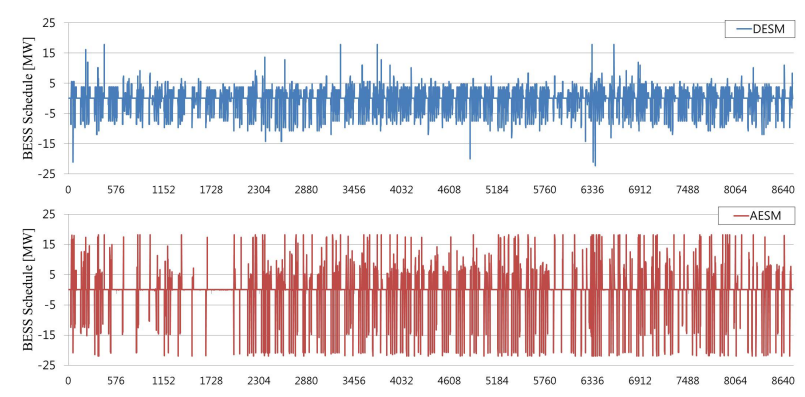

(a) Main Land

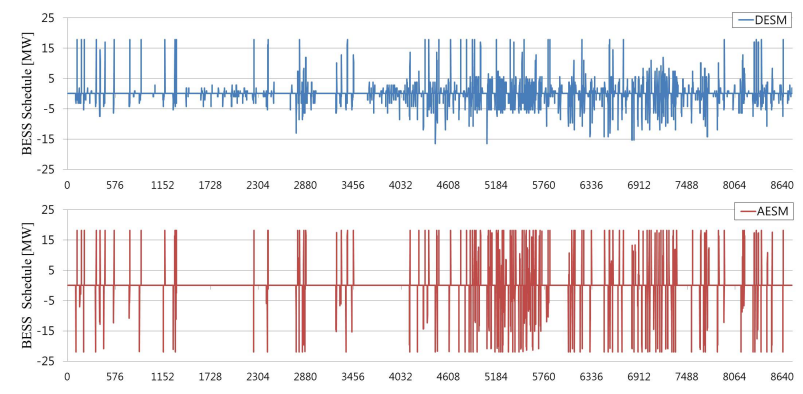

(b) Jeju Island

Fig. 5. Hourly BESS schedules for energy arbitrage by DESM and AESM based on MCPs for a year of 2013

and AESM for the energy arbitrage in 2013 for Main Land and Jeju Island. It is more frequently scheduled in Main Land rather than Jeju Island because the MCP differences in Main Land are generally larger than those in Jeju Island. 


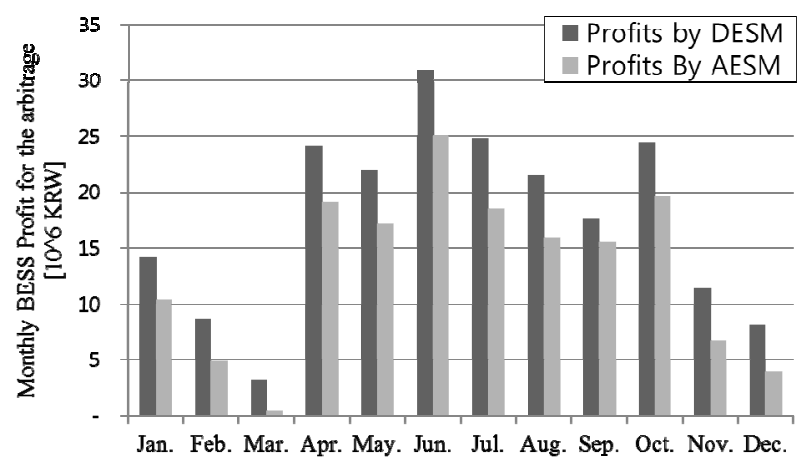

(a) Main Land

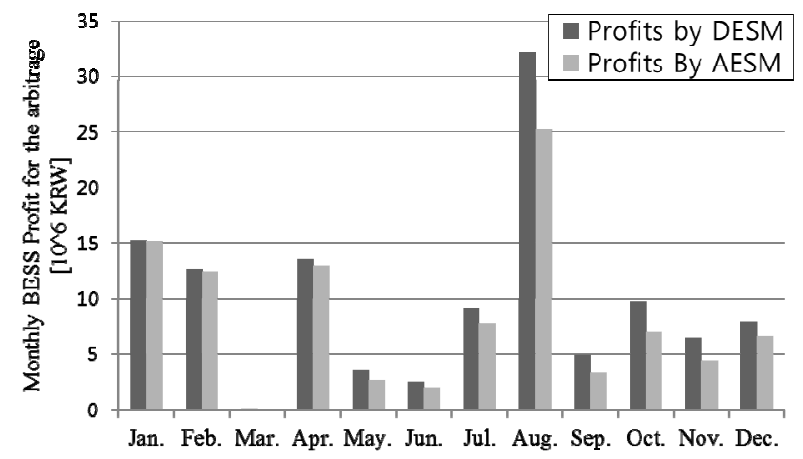

(b) Jeju Island

Fig. 6. Monthly BESS profits from the energy arbitrage by DESM and AESM

And the number of operation days by DESM is more than the days by AESM in both systems. Comparing with AESM, the operation schedule by DESM is generally maintaining low charging and discharging output power levels with the high efficiency range, except when particularly sudden high or low prices are occurred. On the contrary, the scheduled charging and discharging powers by AESM are almost rated values at most scheduled time intervals.

Table 2 is a summary of the operation results for the arbitrage used by AESM and DESM with Li-ion BESS in Main Land and Jeju Island. With 40MWh and 20MW of BESS, AESM make BESS use high powers for schedule with $14.86 \mathrm{MW}$ and $5.40 \mathrm{MW}$ of average charging and discharging power. However, the average scheduled powers by DESM are only $5.87 \mathrm{MW}$ and $3.11 \mathrm{MW}$ under charging and discharging respectively in Main Land.

\subsubsection{Benefits for DESM and AESM}

Fig. 6 shows the monthly profits from the results of operation schedule by DESM and AESM for the energy arbitrage application. In both Main Land and Jeju Island, profits by DESM are greater than those by AESM in every month. Annual energy arbitrage profits by DESM are increased 34.4\% and 18.4\% than AESM in Main Land and Jeju Island, respectively. In particular, even if the small
Table 3. Summary of BESS operation for arbitrage by AESM and DESM

\begin{tabular}{c|c|c|c|c}
\hline Power Grid & \multicolumn{2}{|c|}{ Main Land } & \multicolumn{2}{c}{ Jeju Island } \\
\hline Operation Model & AESM & DESM & AESM & DESM \\
\hline Scheduled Days & 213 & 284 & 109 & 211 \\
\hline $\begin{array}{c}\text { Total Profit } \\
{\left[10^{\wedge} 3 \cdot \mathrm{KRW}\right]}\end{array}$ & 157,128 & 211,104 & 99,681 & 118,035 \\
\hline
\end{tabular}

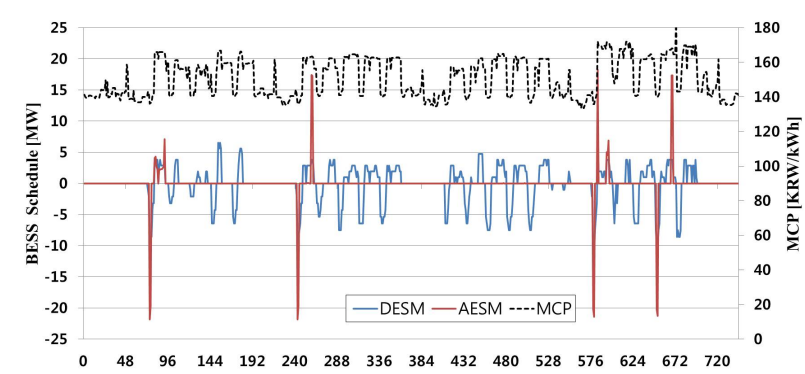

(a) Main Land

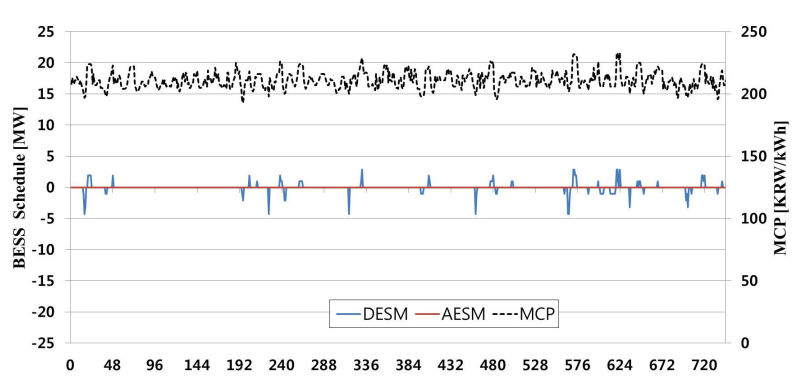

(b) Jeju Island

Fig. 7. BESS operation schedule for energy arbitrage by DESM and AESM in March 2013

MCP differences occur in March, DESM generates the profits of 3.24 and 0.18 million KRW while 0.43 million KRW and zero by AESM in Main Land and Jeju Island, respectively.

Fig. 7 shows the schedules of both systems in March. There can be little opportunity to arbitrage with BESS because this month generally has small daily MCP differences. By DESM, 14 days of charging/discharging operation are occurred while only 4 operating days are occurred for AESM in Main Land. In Jeju Island 14 days are scheduled by DESM with low C-rates of BESS and none is occurred by AESM.

Table 3 summarizes the results of BESS operation by AESM and DESM in Main Land and Jeju Island. By DESM, total operation days in 2013 are increased by $33.3 \%$ and $93.6 \%$, and also total profits are expected more of $34.4 \%$ and $18.4 \%$ comparing with AESM in Main Land and Jeju Island, respectively.

\section{Conclusions}

A dynamic efficiency operation scheduling model of 
BESS corresponding to charging and discharging power output has been developed in this paper. The algorithm has been formulated as mixed integer linear programming and compared with the conventional average efficiency operation algorithm. Two algorithms are tested for the real power systems of South Korea using historical demand and MCP. The results for peak shaving and energy arbitrage by the two models have shown that the developed dynamic efficiency scheduling model is more precise than the model using average value. Although this paper the developed advanced algorithm with variable efficiency of BESS, it is necessary in future to develop more an accurate algorithm considering the efficiency influenced by BESS characteristics of state of charge.

\section{Nomenclature}

\section{Constants}

$L_{j} \quad$ The number of steps of charging efficiency function of BESS-j

$M_{j} \quad$ The number of steps of discharging efficiency function of BESS-j

$\eta_{j}^{c, \ell} \quad$ Charging efficiency corresponding to $\ell$ power section of BESS-j

$\eta_{j}^{d, m} \quad$ Discharging efficiency corresponding to $m$ power section in BESS-j

Cycle $_{j}$ The cumulated number of cycles to limit charging and discharging energy of BESS-j during a schedule period

$D_{t} \quad$ Estimated demand at time interval- $t$

$R_{t} \quad$ Energy price at time interval- $t$

$S O C_{j}^{o} \quad$ Initial state of charge of BESS- $j$

$S_{j} C_{j}^{\max } \quad$ Maximum state of charge limit of BESS- $j$

$S O C_{j}^{\min } \quad$ Minimum state of charge limit of BESS- $j$

$S O C_{j, T}^{\text {low }} \quad$ Low bound of the final state of charge of BESS $-j$ in an operating period

$S O C_{j, T}^{\text {upper }}$ Upper bound of the final state of charge of BESS- $j$ in an operating period

\section{Variables}

$D_{t}{ }^{\prime} \quad$ Adjusted demand considering operation schedules of BESSs at time interval- $t$

$D^{\max } \quad$ Adjusted peak demand considering operation schedules of BESSs for an operating period

$e p_{j, t}^{c} \quad$ Charging power of BESS-j from the power grid at time interval- $t$

$e p_{j, t}^{d} \quad$ Discharging power of BESS-j into the power grid at time interval- $t$

$E P_{j, t}^{c} \quad$ Internal side charging power of BESS- $j$ at time interval- $t$

$E P_{j, t}^{d} \quad$ Internal side discharging power of BESS-j at time interval- $t$

$q_{j, t}^{c, \ell} \quad$ Charging power of BESS-j corresponding to $\eta_{j}^{c, \ell}$ at time interval- $t$

$q_{j, t}^{d, m} \quad$ Discharging power of BESS- $j$ corresponding to $\eta_{j}^{d, m}$ at time interval- $t$

$S O C_{j, t} \quad$ State of charge of BESS- $j$ at time interval- $t$

$v_{j, t}^{c} \quad$ Binary variable which is equal to 1 if BESS- $j$ is under charging at time interval- $t$

$v_{j, t}^{c, \ell} \quad$ Binary variable which is equal to 1 if BESS-j's charging power is $q_{j, t}^{c, \ell}$ at time interval- $t$

$v_{j, t}^{d} \quad$ Binary variable which is equal to 1 if BESS- $j$ is under discharging at time interval- $t$

$v_{j, t}^{d, m} \quad$ Binary variable which is equal to 1 if BESS-j's discharging power is $q_{j, t}^{d, m}$ at time interval- $t$

\section{References}

[1] Sandia National Laboratories, DOE/EPRI 2013 Electricity Storage Handbook in Collaboration with NRECA (SAND2013-5131), July 2013.

[2] D. Maly and K. Kwan, "Optimal battery energy storage system(BESS) charge scheduling with dynamic programming", IEE Proc. Science, Measurement and Technology, Vol. 142, Issue 6, pp. 453-458, Nov. 1995

[3] Oudalov, A., Cherkaoui, R., and Beguin, A., "Sizing and Optimal Operation of Battery Energy Storage System for Peak Shaving Application", in Proc. IEEE Lausanne Power Tech, pp. 621-625, Jul. 2007.

[4] R. Sioshansi, P. Denholm, T. Jenkins, and J. Weiss "Estimating the value of electricity storage in PJM: Arbitrage and some welfare effects", Energy Economics, Vol. 31, Issue 2, pp. 269-277, Mar. 2008.

[5] J. Garcia-Gonzalez, R. M. R. de la Muela, L. M. Santos, and A. M. Gonzalez, "Stochastic joint optimization of wind generation and pumped-storage units in an electricity market", IEEE Trans. Power System, Vol. 23, Issue 2, pp. 460-468, May 2008.

[6] Z. Shu and P. Jirutitijaroen, "Optimal sizing of energy storage system for wind power plants," in Proc. IEEE Power Engineering Society General Meeting, pp. 1-8, Jul. 2012.

[7] C. Lo and M. Anderson, "Economic dispatch and optimal sizing of battery energy storage systems in utility load-levelling operations", IEEE Trans. Energy Conversion, Vol. 14, Issue 3, pp. 824-829, Sep.1999.

[8] S. Grillo, M. Marinelli, S. Massucco, and F. Silvestro, "Optimal management strategy of a battery-based storage system to improve renewable energy integration in distribution Networks", IEEE Trans. Smart Grid, Vol. 3, Issue 2, pp. 950-958, Jun. 2012.

[9] M. S. Kandil, S. A. Farghal, and N. E. Hasanien, "Optimum operating policy for energy storage for an interconnected power system”, IEE Proc. Generation, Transmission and Distribution, Vol. 137, Issue 4, pp. 291-297, Jul. 1990.

[10] T. Y. Lee and N. Chen, "Effect of the battery energy 
storage system on the time-of-use rates industrial customers," IEE Proc. Generation, Transmission and Distribution, Vol. 141, Issue 5, pp. 521-528, Sep. 1994.

[11] Eunsung Oh, Sung-Yong Son, Hyemi Hwang, JongBae Park, Kwang Y. Lee, "Impact of Demand and Price Uncertainties on Customer-side Energy Storage System Operation with Peak Load Limitation", Electric Power Components and Systems, Vol. 43, Issue 16, pp. 1872-1881, Aug. 2015.

[12] F. Mohamed, "Microgrid modelling and online management," Ph.D. dissertation, Helsinki Univ. of Tech., Helsinki, Finland, 2008.

[13] C. Chen, S. Duan, T. Cai, B. Liu and G. Hu, "Optimal Allocation and Economic Analysis of Energy Storage System in Microgrids", IEEE Trans. Power Electronics, Vol. 26, Issue 10, pp. 2762-2773, Oct. 2011.

[14] E. M. Krieger and C. B. Arnold, "Effects of undercharge and internal loss on the rate dependence of battery charge storage efficiency", Journal of Power Sources, Vol. 210, pp. 286-291, Jul. 2012.

[15] Christen, Thomas, and Martin W. Carlen, "Theory of Ragone plots", Journal of power sources, Vol. 91, Issue 2, pp. 210-216, Dec. 2000.

[16] Zhou, Chengke, et al., "Modeling of the cost of EV battery wear due to $\mathrm{V} 2 \mathrm{G}$ application in power systems", IEEE Trans. Energy Conversion, Vol. 26, Issue 4, pp. 1041-1050, Dec. 2011.

[17] Korea Power Exchange, http://www.kpx.or.kr, Dec 12 th 2015.

[18] H. Qian, J. Zhang, J. S. Lai, W. Yu "A high-efficiency grid-tie battery energy storage system", IEEE Trans. Power Electronics, Vol. 26, Issue 3, pp. 886-896, Mar. 2011.

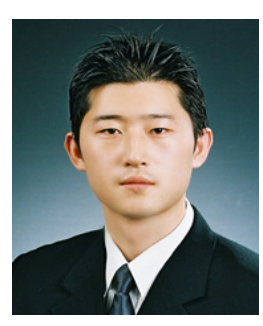

Yong-Gi Park He received B.S., M.S., and Ph.D. degrees from Konkuk University, Korea, in 2005, 2009, and 2014 respectively. For 2005-2007, he was with Samsung Electronics Corporation. Since 2014, he has been with Research Center for Innovative Electricity Market Technology, Konkuk University, Korea. His research interest includes optimal operation and planning of power system, and electricity market analysis.

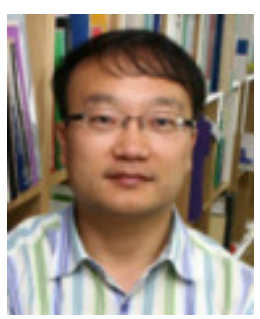

Chang-Wan Kim He received a Ph.D. in NVH analysis by newly developing Automated Multilevel Substructuring (AMLS) method from the University of Texas at Austin. AMLS is now being used in all automobile industries for $\mathrm{NVH}$ analysis. He previously worked in NASTRAN developer in USA and

RecurDyn in Korea. He has over 80 paper publications along with several invited papers and presentations. Now, his research is specialized in multiphysics analysis by integrating CFD-MBD-FEM coupling technology.

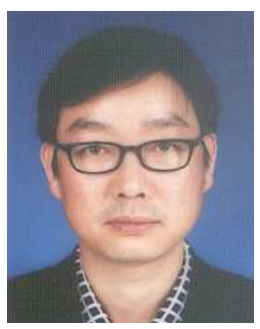

Jong-Bae Park He received B.S., M.S., and Ph.D. degrees from Seoul National University in 1987, 1989, and 1998, respectively. For 1989-1998, he was with Korea Electric Power Corporation, and for 1998-2001 he was an Assistant Professor at Anyang University, Korea. For 2006-2008, he was a guest researcher of EPRI, USA. From 2001, he has been with Electrical Engineering Department at Konkuk University as Professor. His major research topics include power system operation, planning, economics, and markets. 